\title{
EL APLAUSO AL PRESIDENTE AZNAR: ESTRUCTURAS SINTÁCTICO- PRAGMÁTICAS QUE LO PROPICIAN
}


Entre las incidencias (protestas, risas, rumores, etc.) que se suman en un discurso a la palabra del orador, está el aplauso. En este artículo, tras proponer una clasificación de los componentes verbales, paraverbales y kinésicos que propician dicho aplauso, analizaremos el grupo más frecuente que hemos encontrado entre los verbales: aquellos que dependen de estructuras sintáctico-pragmáticas (serie enumerativa, refuerzo argumentativo, posicionamiento y contraste). El corpus del que hemos partido consta de los discursos de José María Aznar emitidos en los debates del estado de la nación en que participó como presidente del gobierno español (1997-2003).

Palabras Clave: discurso político, oralidad, aplauso, mecanismos propiciadores del aplauso

A round of applause is one of the many incidents (e.g. protests, laughs, murmurs) that may occur when the speaker is giving the speech. Drawing on a classification of the verbal, paraverbal and kinesic components that motivate a round of applause, this paper analyzes the most frequent verbal type, namely, that which depends on syntactico-pragmatic structures (i.e. enumerative listing, argumentative reinforcement, positioning, and contrast). The corpus of analysis used in this paper consists of the speeches delivered by José María Aznar at the Debate of the State of the Nation as president of the Spanish government (1997-2003).

KEY wORDS: political discourse, orality, applause, motivating devices

RECEPCIÓN:

ACEPTACIÓN: 


\title{
EL APLAUSO AL PRESIDENTE AZNAR: ESTRUCTURAS SINTÁCTICO- PRAGMÁTICAS QUE LO PROPICIAN ${ }^{1}$
}

\author{
Luis Cortés Rodríguez \\ (Universidad de Almería/ CySOC)
}

\section{Cuestiones previas}

1.1. Ya hemos hablado en un artículo anterior (Cortés, 2015) de lo que entendemos por incidencias; son manifestaciones que se suman en un discurso a la palabra de quien en ese momento tiene el turno. Para contento o para pesar de este, tales actuaciones vienen a formar parte del hecho discursivo; es la otra voz, la de un sector de los interlocutores que sienten la necesidad de hacer llegar su opinión mediante aplausos, rumores, risas, protestas o voces individuales; salvo los primeros, los aplausos, que siempre serán de aprobación, las otras actuaciones, como veremos al analizarlas, podrán ser de aprobación o desaprobación. A tales incidencias, vendrá

\footnotetext{
${ }^{1}$ Este trabajo forma parte de proyecto FFI2012-31699, "Los debates sobre el estado de la nación (1983-2011). Análisis pragmalingüístico”, concedido por el Ministerio de Economía y Competitividad de España.
} 
a unirse la voz de la presidencia ${ }^{2}$ cuando solicite el silencio de la cámara en general o de algún diputado en particular (apartado de silencios). De todas estas manifestaciones, solo la propiciación del aplauso ha merecido la atención de los estudiosos, aunque tal interés haya sido hasta ahora escaso y por parte, generalmente, de psicólogos sociales. ${ }^{3}$

1.2. El aplauso en los debates en torno al estado de la nación (en adelante, DEN $)^{4}$ se ha de ver como una muestra de identidad de grupo, por medio del cual se elogia lo que dice un portavoz; como tal actividad, señaló Bull (2000), tal aplauso requiere la coordinación del citado grupo si se pretende que sea eficaz. La acción del aplauso, como decíamos, es la única incidencia que invariablemente se hace en señal de aprobación o entusiasmo; esta circunstancia puede venir más o menos propiciada por el tema o la idea manifestada, la cual, a veces, se podrá potenciar a través de mecanismos sintáctico-pragmáticos como el refuerzo argumentativo o la serie enumerativa, por citar los dos más empleados. Al ser un acto de aprobación, es normal que el político busque en

\footnotetext{
${ }^{2}$ No consideramos entre tales incidencias la actuación protocolaria de la presidencia de dar la palabra y de agradecer su intervención al final al político de turno.

${ }^{3}$ En español, véase Fernández García (1998). En inglés, Atkinson (1984, 1985, 1994), Bull (2000, 2006, 2012), Bull y Noordhuizen (2000), Bull y Wells (2002), Grady y Potter (1985), Heritage y Greatbatch (1986); Ilie (2001, 2004).

${ }^{4}$ Los DEN se celebran, anualmente, en España y generan siempre un gran interés, pues Gobierno y oposición abordan los asuntos políticos más importantes del país. Instaurados por el socialista Felipe González, en 1983 —en la II Legislatura-, su celebración es anual, y desde entonces hasta nuestros días solamente se han dejado de llevar a cabo en seis ocasiones, todas ellas por la convocatoria de elecciones generales.
} 
momentos clave de sus discursos la ayuda de ciertos mecanismos en su búsqueda del aplauso. El análisis de estos es el punto de interés de este artículo.

Bien es verdad que ni tales mecanismos cumplen en muchas ocasiones su objetivo, el aplauso, aunque lo pretendan, ni este, en otras ocasiones, se puede explicar a través de ellos, especialmente cuando no ocurre en un punto de terminación. Bull (2000), ante ejemplos de este último tipo, propuso diferenciar dos modos distintos de aplausos en los discursos políticos: los demandados y los no demandados; los primeros son los que vienen propiciados por tales mecanismos; ${ }^{5}$ el orador los usará para indicar cuándo y dónde los aplausos son apropiados. Los no demandados son aplausos que ni responden a estos mecanismos ni, en ocasiones, están sincronizados con el discurso; por ejemplo, pueden iniciarse por una pequeña parte de la audiencia $y$, con retraso señalado, ser seguidos por el resto de partidarios. Parece claro que un discurso con puntos 'marcados', que sirvan para la propiciación del aplauso, va a aumentar, en principio, la probabilidad de su éxito, pues se crea la conciencia en cada oyente de que puede comenzar a aplaudir con la convicción de que todos los demás van a hacer lo mismo. Esto requiere ciertas aptitudes por parte del orador,

\footnotetext{
${ }^{5}$ Entre las clasificaciones de mecanismos, la más conocida es la de Heritage y Greatbatch (1986: 122), quienes separaron los aplausos suscitados por el contenido de los suscitados por mecanismos retóricos; entre estos establecieron siete procedimientos: contrast, list, puzzle-solution, headline-punch line, combination, position taking y pursuit. El trabajo, basado en 476 discursos políticos, mostró que casi el $70 \%$ de los aplausos fue precedido de un mecanismo de este tipo, independientemente de ideologías y demás.
} 
aptitudes de las que, por ejemplo, el expresidente español J.M. Aznar (en adelante, JMA) no estaba muy dotado.

En síntesis, determinados tipos de mecanismos retóricos, además de la importancia de los temas, bien usados pueden servir de 'llamada' para que todos actúen al mismo tiempo y se sepa cuándo empezar el aplauso. Además, estos, al ir próximos a un punto de finalización del enunciado, suelen ir acompañados de cambios de gestualización, aumento del volumen de la elocución y del ritmo, pausas diferentes, curvas entonativas, etc. ${ }^{6}$ Es una cualidad del buen orador transmitir estos aspectos a la audiencia. Cuando se retrasa el aplauso o cuando no es entendido por el orador, tal aplauso suele fracasar por motivos diversos.

\section{Aspectos Metodológicos}

\subsection{Corpus}

En este artículo pretendemos el análisis de los aplausos recibidos por JMA, durante sus dos mandatos como presidente del gobierno español, en los discursos iniciales emitidos en los DEN celebrados en los años 1997, 1998, 1999, 2001, 2002 y 2003 (legislaturas VI y VII). Para ello, nos valdremos de

\footnotetext{
${ }^{6}$ En resumen, podemos decir con Heritage y Greatbatch (1986: 116) que un buen orador no ha de olvidar, sino todo lo contrario: a) un especial énfasis (entonación, el ritmo o el volumen, así como el comportamiento no verbal) que muestre a la audiencia que se trata de un mensaje de importancia (interés temático) digno de preparar el aplauso, b) una estructura tal que permita al público saber con antelación cuándo va a terminar, para así poder aplaudir en el momento 'justo'.
} 
las transliteraciones aparecidas en el Diario de sesiones, así como de los vídeos correspondientes. En el cuadro siguiente podemos observar la duración de sus discursos, el número de aplausos por año y su porcentaje total por minuto. Cfr. cuadro $n^{\circ} 1$ :

\begin{tabular}{|c|c|c|c|c|c|c|c|}
\hline \multicolumn{7}{|c|}{$\begin{array}{c}\text { Cuadro }{ }^{\circ} 1 \text {. Aplausos en los discursos iniciales } \\
\text { del presidente Aznar en los DEN }\end{array}$} & \multirow[b]{2}{*}{ Total } \\
\hline Año & 1997 & 1998 & 1999 & 2001 & 2002 & 2003 & \\
\hline Aplausos & 1 & 17 & 7 & 3 & 3 & 4 & 35 \\
\hline Duración & 1h. 09'14" & 1h. 10'37" & $49^{\prime} 15^{\prime \prime}$ & 1h. $02^{\prime} 28^{\prime \prime}$ & 00hs.57'54" & 00hs.58'.01" & 6h08'23" \\
\hline$\%$ por minuto & & & & & & & 10.387 \\
\hline
\end{tabular}

JMA fue un presidente muy poco aplaudido, especialmente si lo comparamos con Rodríguez Zapatero, su sucesor en las dos siguientes legislaturas, cuyos datos en este sentido fueron tan diferentes. ${ }^{7}$

Como se puede observar en el cuadro no 1 , el líder popular en sus seis intervenciones, con una duración total de $6 \mathrm{~h}$ 08'23", fue aclamado treinta y cinco veces, lo que hace que estas se produjeran cada 10'22", porcentaje que no es comparable, por ejemplo, con el de su sucesor en la presidencia, aplaudido cada 3’49”. Es más, en su discurso de 1998,

${ }^{7}$ Los datos del líder socialista como presidente fueron:

\begin{tabular}{|c|c|c|c|c|c|c|c|}
\hline \multicolumn{7}{|c|}{ Cuadro n'2. Aplausos en los discursos iniciales } & \\
\hline Año & 2005 & 2006 & 2007 & 2009 & 2010 & 2011 & Total \\
\hline Aplausos & 38 & 32 & 47 & 27 & 13 & 7 & 164 \\
\hline Duración & $01 \mathrm{~h} .30^{\prime}$ & $01 \mathrm{~h} .122^{\prime} 27^{\prime \prime}$ & $01 \mathrm{hs} .15^{\prime} 30^{\prime \prime}$ & $01 \mathrm{hs} .01^{\prime} 31^{\prime \prime}$ & $01 \mathrm{hs} .06^{\prime} 01^{\prime \prime}$ & $01 \mathrm{hs} .06^{\prime} 15^{\prime \prime}$ & $\mathbf{6 h 4 5} \mathbf{6 0}$ \\
\hline$\%$ por minuto & & & & & & & $\mathbf{2 . 4 6}$ \\
\hline
\end{tabular}


el más ovacionado, seis de esos aplausos fueron en buena parte propiciados por los rumores de desaprobación de los diputados de la oposición; los rumores se intentan acallar mediante aplausos. Algo parecido sucede en dos ocasiones, ese mismo año, con la risa, que también se repite en 2003 precediendo al aplauso.

Como veremos más adelante, de esas treinta y cinco ocasiones, en once se dio un solo elemento propiciador; en quince, se dieron dos conjuntamente y en nueve ocasiones, tres elementos. Por tanto, nuestro corpus constará de treinta y cinco aplausos y sesenta y ocho mecanismos propiciadores. En los próximos apartados intentaremos analizar algunos aspectos de estos mecanismos y contenidos.

\subsection{Mecanismos de propiciación del aplauso}

2.2.1. El análisis llevado a cabo en corpus más amplios nos sirvió para establecer una tipología de procedimientos propiciadores del aplauso en este tipo de discurso político. Hemos establecido los siguientes apartados, en los cuales mostramos entre paréntesis los casos encontrados en Aznar:

\section{COMPOnEnTE Verbal}

\section{Procedente del discurso del orador}

Aspectos temáticos

\section{- Anuncio}

- Mención de personas o instituciones (3)

- Resultado de acciones previas (2)

- Tema en sí (6) 
Aspectos dependientes de estructuras sintáctico-pragmáticas

- Serie enumerativa (17)

- Refuerzo argumentativo-conclusivo: consecuencia, justificación y reformulación (16)

- Posicionamiento (7)

- Contraste (6)

Procedente de otros participantes del discurso

- Protestas

- Voces (3)

- Petición de silencio

COMPONENTES PARAVERBAL Y KINÉSICO

Procedente del discurso del orador

Procedente de otros participantes del discurso

- Risas (1)

- Rumores (7)

A la hora de hablar de estos mecanismos, creemos necesario hacer algunas aclaraciones antes de entrar en el objeto de este estudio: los aspectos sintáctico-pragmáticos propiciadores del aplauso. En primer lugar, hemos de señalar la ausencia en los corpus analizados de aplausos que hayan estado motivados únicamente por el componente kinésico; esto no quiere decir que no se hayan dado en otros corpus (imaginemos cualquier gesto de triunfo del orador - por ejemplo, el pulgar hacia arriba- y los posteriores aplausos de reacción de sus señorías). También conviene concretar 
que tales procedimientos pueden o bien darse aisladamente (en once casos) o, lo que es más frecuente, en confluencia con alguno más (los veinticuatro restantes); la tercera aclaración nos lleva a recordar lo reducido del corpus de JMA, y esto hace que en algunos apartados de la tipología arriba señalada no se haya encontrado ejemplo alguno; finalmente, no hemos de olvidar que cualquiera que sea el tipo de aspecto analizado, junto a él, imbricado con el mecanismo, siempre estará la alteración de lo prosódico. Por tanto, la presente clasificación no se ha de interpretar como una separación entre los varios elementos que suelen subyacer en el aplauso, sino como un intento de poner algo de orden en el descubrimiento de esos distintos mecanismos que lo propician. A modo de ejemplo, veamos este enunciado de Aznar [2001]:

(1) Señoras y señores diputados, yo no sé si bajar impuestos es de izquierdas, de centro o de derechas, pero me alegro de que ya no estemos tan solos en la defensa de las virtudes de bajar los impuestos y de reducir los tramos. (Aplausos.-Rumores) ${ }^{8}$ [Aznar, 2001]

El contenido, la bajada de impuestos, es siempre bien recibido y, por tanto, forma parte de los 'cualificados' para el aplauso: se trata de alabar conquistas del Gobierno presidido por el orador; en este caso, la bajada de impuestos

\footnotetext{
${ }^{8}$ En verdad lo que se produce es primero unos rumores lejanos y breves, tras la serie enumerativa, y posteriormente los aplausos, sin que haya relación, creemos, entre ambos hechos.
} 
no solo habrá de ser bien recibida por los correligionarios, sino por todos los españoles en general. A ese contenido, merecedor ya de aplauso, se unirán otros mecanismos verbales y no verbales. Así, el inicio, tras una pausa más larga de lo normal, se hace mediante un vocativo de advertencia con el que se parece anunciar el interés de lo que se va a decir; posteriormente, se recurre al posicionamiento del presidente, se sigue con la serie enumerativa, del tipo lineal (de izquierdas, de centro o de derechas) y, finalmente, se cierra con otra serie enumerativa, esta paralelística; tales mecanismos, las series enumerativas y el posicionamiento del orador son dos de las cuatro estructuras, como veremos, más empleadas por Aznar. Al mismo tiempo, hay tanto un ligero aumento del énfasis y el volumen de la voz en ambas series enumerativas como un cambio de velocidad, que lleva al presidente popular a aumentar su flexibilidad en lo dicho. En otros políticos, sin embargo, hubiera sido normal que estos aspectos se acompañaran de movimiento de brazos, de gestos, etc., lo que sin embargo no se dio en el político que analizamos, quien mantuvo, como casi siempre, sus palmas de las manos sobre el atril y su mirada alternando el papel y el público, pero con prioridad en aquel.

Aun siendo conscientes, por tanto, de esta sincronía discursiva, hemos querido establecer la citada clasificación, cuyos tipos se acompañarán constantemente de los aspectos no verbales, especialmente kinésicos y paraverbales.

2.2.2. En el cuadro siguiente, cuadro no 3 , podemos conocer detalladamente el tipo de propiciador que se dio en cada uno de los treinta y cinco aplausos, así como su 
forma de aparición: un mecanismo solo (11 casos); dos mecanismos juntos (15 casos) o tres (9 casos):

\section{Cuadro no 3. Aparición de los mecanismos de propi- ciación del aplauso}

\begin{tabular}{|c|c|c|c|c|c|c|c|c|}
\hline AÑO & \multicolumn{4}{|c|}{  } & \multicolumn{2}{|l|}{ AÑo } & \multicolumn{2}{|l|}{9 TRIPLES } \\
\hline 1997 & Acc. previas & 1998 & Consec. & Rumores & 1998 & Ser.enu.par. & Contraste & Voces \\
\hline \multirow[t]{8}{*}{1998} & Consecuenc. & & Contraste & Rumores & & Menc. personas & Posicion. & Voces \\
\hline & Consecuenc. & & Acc. previas & Rumores & & Menc. personas & Posicion. & Rumores \\
\hline & Rumores & & Tema & Rumores & & Contraste & Ser.enu.par. & Voces \\
\hline & Tema & & Tema & Rumores & & Reformulación & Contraste & Ser.enu.par. \\
\hline & Tema & 1999 & Justificac. & Ser.enu.l. & 2001 & Ser.enu.l. & Posicion. & Ser.enu.par. \\
\hline & Tema & & Menc. Pers. & Posicionam. & & Contraste & Posición & Ser.enu.par. \\
\hline & Tema & & Ser.enu.par. & Ser.enu.l. & 2002 & Contraste & Posicion. & Ser.enu.par. \\
\hline & Consecuenc. & & Consecuenc. & Ser.enu.l. & & Consecuenc. & Posicion. & Ser. enu.l \\
\hline \multirow[t]{6}{*}{1999} & Consecuenc. & & Justificación & Ser.enu.l. & & & & \\
\hline & Consecuenc. & 2001 & Consecuenc. & Ser.enu.par. & & & & \\
\hline & & 2002 & Consecuenc. & Ser.enu.par. & & & & \\
\hline & & 2003 & Justificac. & Ser.enu.par. & & & & \\
\hline & & & Consecuenc. & Risas & & & & \\
\hline & & & Justificac. & Ser.enu.l. & & & & \\
\hline
\end{tabular}

Se puede ver que fueron seis los ejemplos suscitados por el tema en sí, todos ellos en $1998 .{ }^{9} \mathrm{Al}$ ser discursos emitidos por los presidentes ${ }^{10}$ en los DEN, es lógico que la alusión a un 'éxito' del Gobierno sea, en ocasiones, motivo

\footnotetext{
${ }^{9}$ Hay detalles como este que hacen pensar en autores distintos de los discursos, pues en los tres DEN de esta legislatura no hubo marcadas diferencias económicas, sociales, etc.

${ }^{10}$ Contrariamente, en los líderes de la oposición la cuestión más tratada y aplaudida será la crítica a sus rivales políticos, a otros partidos políticos, especialmente al partido del Gobierno, a sus actuaciones, a su presidente, etc.
} 
de aplauso; por ejemplo, cuando el presidente Aznar habla de las reformas llevadas a cabo con las tarifas eléctricas:

(2) Me van a permitir un ejemplo: si hubiéramos continuado con el marco legal estable, las tarifas eléctricas hubieran subido un 4 por ciento anual. Gracias a las reformas que hemos emprendido, se han reducido en dos años más de un 7 por ciento. (Aplausos.) [Aznar, 1998]

Está claro que solo gracias a la labor del gobierno que preside a los españoles nos hemos ahorrado un dinero considerable; todos hemos de estar agradecidos con su actuación. Y para presentar su idea el presidente de vale de un argumento basado en datos; Aznar presenta una información irrebatible: datos mediante informaciones que se presumen como ciertas o válidas; tal argumentación suele tener una efectividad concreta a la hora de convencer a los receptores. Igualmente ocurre cuando se refiere a la seriedad de sus proyectos:

(3) Siempre he estado convencido de que la fortaleza de una democracia la da, más allá de las personas, la seriedad de los proyectos políticos y la solidez de los partidos. (Aplausos.) [Aznar, 2003]

Su argumento, en esta ocasión, está basado en valores, en lo convencionalmente aceptado: seriedad y solidez son dos virtudes de las que está convencido cualquier receptor.

También, al reparar en el cuadro, cabe reseñar los tres casos en los que la mención positiva de personas, de grupos o instituciones, de las que se encomia su actuación, conduce, junto a otros mecanismos (sintáctico-pragmáticos), 
al aplauso. Las menciones negativas al enemigo formarían parte de los aplausos emitidos por el contenido, pues su origen es diferente. En los casos que nos ocupan, los aludidos han sido personas; por ejemplo, soldados en misiones de paz en el exterior:

(4) En estos momentos, más de 3.500 mujeres y hombres de nuestros ejércitos se encuentran en distintas misiones defendiendo nuestros valores en el exterior. Quiero dedicarles un especial recuerdo desde esta tribuna. (Aplausos.) [Aznar, 1999]

trabajadores autónomos:

(5) Existen en España millones de autónomos que trabajan solos y a los que quiero dirigirme de una manera muy singular para decirles que este es el momento de tomar la iniciativa y que esta es la ocasión de asumir nuevos objetivos. (Varios señores diputados: ¡Muy bien!-Aplausos.) [Aznar, 1998]

y una institución: el Ministerio Fiscal:

(6) Quiero, en todo caso, reiterar mi respeto a la autonomía del Ministerio Fiscal, en el que depositamos la confianza más plena para el cumplimiento de su papel constitucional de defensa del interés público. (Aplausos.) [Aznar, 1998]

En todas las ocasiones, aunque no hay cambios en la gestualidad sí se ve un aumento del volumen de la elocución y del ritmo, pausas diferentes y curvas entonativas ligeramente más señaladas. 
Una última mirada al cuadro, antes de pasar al análisis de los aspectos sintáctico-pragmáticos, nos permite observar la importancia del componente paraverbal y kinésico. A veces, lo dicho por el orador no es aceptado por la oposición, que reaccionará mediante rumores (seis casos) o risas (un caso); en ese momento, como medida para acallar tales manifestaciones, es frecuente que los correligionarios del presidente reaccionen con aplausos. Veamos algunos casos:

(7) Se ha dado ya un primer paso para mejorar esta situación con la ampliación de la cobertura de los contratos considerados marginales. Los tristemente contratos basura han desaparecido de nuestra legislación. (Rumores.-Aplausos.) [Aznar, 1998]

En este ejemplo, a pesar de lo atrayente del tema, no había habido reacción por parte del grupo popular; de hecho, JMA ya había iniciado el enunciado siguiente "El Gobierno ..., inicio que, ante los rumores de sus oponentes, repetirá una vez más "El Gobierno ..."; es en ese momento cuando sus correligionarios, ante la insistencia en el rumor por parte del hemiciclo, emiten el aplauso, que el presidente no interrumpe. $\mathrm{Al}$ ser un aplauso no demandado, hay una falta de sincronización entre discurso y aplauso. ${ }^{11}$ Exactamente igual ocurre en el caso siguiente, donde dicha falta de sin-

11 Hay ejemplos en que no solo se cumplen varios requisitos (inicio con vocativo, serie enumerativa, refuerzo argumentativo, etc.) para el aplauso, sino que Aznar parece esperar dicho aplauso:

Señor presidente, todos sabemos que con el euro desaparece el tipo de cambio como instrumento para conseguir falsas ganancias de competitividad. Por ello, la acción del Gobierno se ha centrado en tres ejes: reformar y libera- 
cronización se percibe al haber iniciado ya el enunciado posterior, "los casi nueve ..." cuando la reiteración de las protestas inclinó al aplauso al grupo popular:

(8) Señorías, la reforma va a bajar el impuesto, especialmente a los que trabajan por cuenta ajena. Es a los que ganan menos de dos millones de pesetas a quienes más se les reduce la factura fiscal. (Rumores.-Aplausos.) [Aznar, 1998]

El dato final es concluyente, pues son la inmensa mayoría de españoles quienes se van a ver favorecidos fiscalmente. En otras ocasiones, como en el suceso que sigue, el aplauso, que se quiere llevar a cabo tras los ligeros murmullos de los rumores, resulta fallido, pues solo lo sigue una minoría de personas: ${ }^{12}$

(9) El Gobierno es consciente de que nuestro esfuerzo investigador, sin renunciar al equilibrio entre la ciencia básica y la aplicada, debe estar al servicio de la empresa y de la sociedad. No me cuesta reconocer que los recursos destinados a I+D son todavía escasos. Pero quiero recordar que, en los dos últimos años, el gasto presupuestario se ha visto incrementado en un 23 y en un

lizar los mercados, modernizar nuestro sector público empresarial y reforzar nuestro sistema de ciencia y tecnología. [Aznar, 1998].

${ }^{12}$ Más fallido resulta por el menor número de aplausos este otro evento, también en Aznar [1998] y sin que precedan los rumores:

Concluida la aprobación de estos planes de cuenca, por demasiado tiempo pendientes, comienza ahora la nueva etapa que culminará con la aprobación del Plan Hidrológico Nacional; plan que está llamado a ser factor decisivo de vertebración de nuestro territorio. (Aplausos.-Rumores.) 
33 por ciento, respectivamente. La tendencia marcada seguirá el próximo año. En este campo, la coordinación vuelve a ser imprescindible. Por mi parte, he querido presidir la Comisión de Ciencia y Tecnología y crear una nueva oficina, radicada en la Presidencia del Gobierno, de apoyo a nuestro sistema de ciencia y tecnología. (Rumores.-Aplausos.)

En la referencia siguiente, no podríamos decir si el Insalud había tenido un plan de actuación anterior en sus veinte años de existencia, pero lo cierto es que el presidente lo afirma y sus oponentes políticos, especialmente quienes habían ostentado el poder en gobiernos anteriores, no pueden estar de acuerdo con esta afirmación, lo que provoca su rechazo mediante rumores, que se intentan acallar con el aplauso:

(10) El incremento presupuestario destinado a la sanidad pública para 1998, que supone un crecimiento de más de $386.000 \mathrm{mi}$ llones de pesetas, dice más que cualquier argumento sobre la autenticidad del compromiso del Gobierno con la mejora de nuestra sanidad pública. Lógicamente, nuestro tercer objetivo, después de aprobarse el informe y quedar asegurada la financiación, fue dotar al Insalud de un plan de actuación, que fue, por cierto, el primero que se conoce en sus 20 años de funcionamiento. (Rumores.-Aplausos.) [Aznar, 1998]

En este otro ejemplo, fue la risa de la oposición la que incitó al aplauso; la idea de que la alta velocidad se empiece a desplegar por toda España no fue bien aceptada por la oposición: 
(11) Debemos ser conscientes de que es precisamente el equilibrio de las cuentas públicas lo que nos permite realizar una inversión tan importante. La red ferroviaria de alta velocidad empieza a desplegarse por toda España. (Risas.—Aplausos.)

No obstante, son las estructuras sintáctico-pragmáticas las que con más frecuencia se dan como propiciadores del aplauso, y de ellas nos vamos a ocupar.

\section{ESTRUCTURAS SINTÁCTICO-PRAgMÁticos}

De los sesenta y ocho aspectos que propiciaron los aplausos a JMA (véase cuadro $n^{\circ} 3$ ), cuarenta y seis dependieron de estructuras sintáctico-pragmáticas; de ellas, solo cinco (todas con relación consecutiva) aparecen como mecanismo único previo al aplauso; veinte comparten la función con otro mecanismo y veintiuna lo hacen junto a dos. En cuanto a su frecuencia, hemos de decir que la serie enumerativa en 17 ocasiones y el refuerzo argumentativo, en 16, son las más empleadas; le siguieron en orden de frecuencia el posicionamiento, en siete, y en seis, el contraste.

\subsection{Serie enumerativa}

En nuestro trabajo sobre la serie enumerativa (Cortés, ed., 2008) difundimos un concepto amplio de este término, que nada tenía que ver con el único tipo citado en los pocos trabajos previos que se habían ocupado de esta cuestión: la 'lista de palabras'. Para nosotros el término es más amplio e 
incluye, junto a las series enumerativas lineales (donde se incluiría la lista de palabras), otro tipo de series enumerativas, que denominamos de estructura paralelística. Tal idea es la que hemos seguido defendiendo en nuestros distintos estudios, pues en ambos modelos de series estamos ante la aparición de varios elementos simétricos y equifuncionales dependientes de una matriz y que suelen cumplir la misma función discursiva de énfasis. ${ }^{13}$

El empleo de este mecanismo es clave no solo como intensificador de razones para reforzar un argumento, sino por su capacidad para facilitar, al menos a priori, la audibilidad e inteligibilidad de un discurso al estar formado por unidades melódicas cortas y repetidas que articulan mejor lo dicho, a la par que lo hace más aprehensible al disponer el interlocutor de varias opciones que le permitan una más fácil descodificación. Aznar lo utiliza en diecisiete ocasiones como propiciador del aplauso. Es sintomático, por ejemplo, que lo emplee junto con otros mecanismos en el último enunciado de cuatro de sus seis discursos:

(12) Señorías, hay que proyectar, planear y debatir juntos acerca de cómo podemos servir mejor las aspiraciones e intereses de los españoles. Nosotros queremos hacerlo, y ese es el sentido y la razón de toda la obra del Gobierno. (Fuertes aplausos.-Rumores.) [Aznar, 1999]

\footnotetext{
${ }^{13}$ En ocasiones no es fácil la separación entre las dos categorías, como ocurre en el caso siguiente:

La paz que anhelamos ha de fundarse en la dignidad humana, en la justicia, en el respeto de los derechos y en las libertades individuales. Esa será la paz que tendrá la fortaleza y la voluntad para ser generosa. (Aplausos.)
} 
(13) Este es mi compromiso ante la Cámara y ante todos los ciudadanos españoles. Muchas gracias, señora presidenta. (Fuertes y prolongados aplausos.) [Aznar, 2001]

(14) Un Gobierno que quiere llegar más lejos en los grandes retos de los españoles: combatir el terrorismo, asegurar el ejercicio de las libertades, fortalecer la vertebración territorial, seguir creando empleo, modernizar nuestro país, incrementar el bienestar y reforzar nuestro papel en el mundo. Los españoles confiamos en nosotros mismos y eso es lo que nos permite plantearnos nuevos retos para nuestro futuro. Somos una sociedad más libre, más fuerte, más desarrollada y más decidida a seguir avanzando, y estos creo que son los verdaderos rasgos distintivos del estado de nuestra nación. Muchas gracias, señora presidenta. (Prolongados aplausos.) [Aznar, 2002]

(15) Yo creo en ese futuro y pienso que todos los españoles, sean cuales sean nuestras ideas y nuestras aspiraciones, podemos sumar energías y esfuerzos para hacer de España un país cada día mejor. Muchas gracias. (Fuertes y prolongados aplausos de los señores diputados del Grupo Popular, puestos en pie.) [Aznar, 2003]

Como vemos todos los cierres terminan con la alabanza de la nueva España, la de esa sociedad que combate el terrorismo, más libre, más moderna, etc. Pero creemos que el más emotivo es el cierre de 2002 (ejemplo n¹4), en el que se recurre en tan breve espacio de tiempo a dos largas series enumerativas cuya prosodia va marcando un final más plausible, en especial la serie segunda, serie lineal, formada por cuatro elementos: libre, fuerte, desarrollada y decidida; 
en su emisión, el presidente modifica el volumen de su voz, la longitud de sus pausas y la velocidad, que se acorta, en aras a enfatizar el contenido de dichos elementos de la serie; es más, todo ello lo acompaña, lo que no es poco en Aznar, del movimiento de la mano derecha señalando al hemiciclo y el ligero movimiento de cabeza. En general, son argumentos basados en descripciones, en los que se acumulan varias razones; si se trata de persuadir sobre la importancia de algo en virtud de varias causas, lo más lógico será describir ese fenómeno, añadiendo detalles que terminen por concluir en la ventaja o desventaja de su aplicación.

Cuantos más elementos y número de niveles tenga la serie, mayor debe ser el interés tanto ornamental como reforzador del mecanismo. Es el caso de esta serie de cuatro elementos, con estructura paralelística y compleja: ${ }^{14}$

(16) Hoy vemos los beneficios de la austeridad, de la flexibilización de los mercados, de la reducción de los impuestos, de la disminución del tamaño del sector público; hoy vemos las ventajas de contar

${ }^{14}$ Es compleja al tener más de un nivel de dependencia (Cortés, 2005: 368):

Hoy vemos los beneficios

de la austeridad,

de la flexibilización de los mercados,

de la reducción de los impuestos,

de la disminución del tamaño del sector público

hoy vemos las ventajas

de contar con una economía

sana $y$

abierta y

de lo mucho que favorece a todos la expansión de la libertad. 
con una economía sana y abierta y de lo mucho que favorece a todos la expansión de la libertad. (Aplausos.) [Aznar, 1999]

El presidente popular, decíamos, fue, según los DEN analizados, poco aplaudido, tanto por la escasez de mecanismos empleados como por la propia actitud del orador. Así, no espera el aplauso tras la serie citada, pues si bien da el ritmo apropiado a cada uno de los elementos integrantes de la compleja serie enumerativa y si bien es un tema cuyo contenido es positivo, el líder popular sigue con la mirada en los papeles y, lo que es más indicativo, no hace pausa alguna al final de la serie para propiciar el aplauso; de hecho, tras iniciarse este tímidamente, sigue con el inicio del enunciado siguiente: "Sobre estas reformas ..." y solo el fortalecimiento de ese aplauso lo lleva a interrumpir dicho enunciado para atender la reacción de sus correligionarios. Por un motivo u otro, la coordinación orador/correligionarios se produce en menos ocasiones de las que cupiera esperar.

La serie por antonomasia es la de tres elementos, la más empleada en cualquier tipo de discurso, también en el político y en el personaje analizado:

(17) No deja de sorprenderme que algunos persistan en mantener el debate político en términos retrospectivos, intentando resucitar tentaciones de particularismos, exclusiones o insalvables fracturas ideológicas (Aplausos.), como si nada hubiera pasado en el mundo y en la sociedad española en las últimas décadas. [Aznar, 1999]

En este ejemplo de serie tripartita, encontramos la falta de sincronización entre palabra y aplauso. Es posible que 
JMA no previera alargar la pausa tras la serie enumerativa (fracturas ideológicas) en espera del aplauso; posiblemente, quienes escribieron el discurso fueron conscientes de la relación serie enumerativa/aplauso, pero no así el presidente, quien, tras la serie, prescinde de pausa alguna y sigue con su discurso, como en otras varias ocasiones: "Como si nada hubiera pasado en el mundo...", enunciado que se interrumpe con el refuerzo del aplauso de sus correligionarios.

También encontramos las series de dos elementos acompañadas de una estructura paralelística con elementos comunes, como ocurre en el ejemplo siguiente de "la+sustantivo+de los":

(18) Siempre he estado convencido de que la fortaleza de una democracia la da, más allá de las personas, la seriedad de los proyectos políticos y la solidez de los partidos. (Aplausos.) [Aznar, 2003]

\subsection{Refuerzo conclusivo-argumentativo}

Este tipo de refuerzo hemos de relacionarlo con la conclusión temática, o sea con el cierre de un tema ya, por lo visto, suficientemente desarrollado aunque falto de un refuerzo final; en este sentido, enmarca al cerrar dicho tema. Pero además de ello, en estos mecanismos se ha de ver el punto de vista del político acerca del tema citado; hemos de ver en ellos el momento elegido para reforzar, culminar y cerrar un determinado tema. La preparación del aplauso mediante tal refuerzo argumentativo es, sin duda, el recurso previo más empleado antes de que este se produzca. En nuestro corpus, dieciséis de las ovaciones han tenido esta disposi- 
ción mediante estructuras que responden a las conexiones semánticas que la Academia (2009: 2361) considera que llevan a cabo conectores consecutivos e ilativos, explicativos, reformuladores, ejemplificativos y recapitulativos, y que nosotros hemos considerado con función de refuerzo argumentativo en todos los casos. Se han establecido tres tipos diferentes de relaciones según el significado: consecutivo, justificativo y reformulador, si bien en la mayoría de ocurrencias los supuestos conectores están implícitos. De ellas, la más utilizada, obviamente, tanto por Aznar como en otros corpus, es la estructura con significado consecutivo (once casos). Se ha de aclarar que cuando hablamos de tal significado lo hacemos en un sentido amplio, ya que no solo abarcará las relaciones sugeridas por los conectores considerados como consecutivos y recapitulativos por la Academia, sino también aquella otra que, más que una consecuencia de lo precedente, va más allá y aprovecha la información previa para introducir una afirmación más rotunda, deducida e inferida de ella por el orador. Mediante la relación consecutiva, en este sentido amplio, parece ser que el político quiere ser más determinante. Aznar la usa en once ocasiones de las dieciséis del refuerzo argumentativo. En general suelen ir apoyando alguna consecución del Gobierno. Veamos algunos ejemplos:

(19) No quiero dejar de destacar la bonificación de las cuotas a la Seguridad Social para empresas que sustituyan a sus empleadas durante el embarazo. Queremos remover los obstáculos a la contratación de mujeres por causa de su maternidad y facilitar la incorporación de nuevos trabajadores durante el período de baja. 
La maternidad debe dejar de ser un factor que penalice el trabajo de la mujer. (Aplausos.) [Aznar, 1998]

El acto argumentativo se separa con una pausa mayor del resto del enunciado y su curva descendente también es más pronunciada. Hay mayor conciencia de cierre temático. El presidente parece esperar el aplauso, lo que se produce de forma coordinada en la mayoría de casos:

(20) En los hospitales se han conseguido nuevas e importantes reducciones de las listas y tiempos de espera: los pacientes con demora superior a nueve meses han pasado de casi 35.000, en junio de 1996, a menos de 1.000, en 1997. La demora media descendió, en ese mismo período de tiempo, de siete a tres meses, y nuestro objetivo es que este año baje a 80 días (Aplausos.) [Aznar, 1998]

(21) Y ello se traduce en ventajas tangibles para todos. Por poner un solo ejemplo, que afecta a un bien tan básico como la vivienda, a comienzos de esta década una familia media tenía que dedicar más de la mitad de su renta a la compra de una vivienda; hoy, ese esfuerzo se ha reducido al 28 por ciento. Sólo durante el último año, esa reducción ha sido de seis puntos. Una familia, con una hipoteca de ocho millones, paga hoy 20.000 pesetas menos al mes que hace dos años. (Aplausos.) [Aznar, 1998]

Solo en dos ocasiones el presidente utiliza este significado y no hace la pausa correspondiente, sino que continúa su discurso, interrumpido inmediatamente con el aplauso de sus correligionarios por el efecto del significado conclusivoargumentativo: 
(22) Nos ha preocupado particularmente el saneamiento del sistema financiero de la Seguridad Social. Hemos cumplido los objetivos de déficit en estos tres años, lo que nos permitirá equilibrar su presupuesto en el año 2000. Gracias a las reformas y al clima de confianza generado, el dinamismo de nuestra economía ha permitido que hoy haya dos millones de cotizantes más a la Seguridad Social que hace tres años. Este es el principal activo que tiene hoy nuestro sistema de Seguridad Social, pero sobre todo es una garantía para ellos y para sus familias. El Pacto de Toledo necesita (Aplausos.-Rumores.) [Aznar, 1999] El Pacto de Toledo necesita $[\ldots]$

JMA, que ha recurrido a un ritmo pausado y a un tono más elevado para referirse a la estructura paralelística de esta serie enumerativa (para ellos y para sus familias), no hace, sin embargo, una pausa más larga tras sus familias para propiciar el aplauso, sino que este se produce cuando ya ha iniciado el enunciado siguiente "El Pacto de Toledo ...", enunciado que luego continuará tras la ovación: "El Pacto de Toledo necesita ...". Parece claro que o el presidente no busca el aplauso o sus correligionarios no encuentran el acierto de la coordinación en las pocas oportunidades en que lo pretenden. Igualmente ocurre con este otro caso:

(23) Esta orientación debe proseguir en los próximos años porque la base de la sociedad de las oportunidades es que nadie se quede en la cuneta. Contar con un pacto de solidaridad en virtud del cual nadie quede excluido y sin una nueva oportunidad es la condición para una sociedad del bienestar que apueste por el futuro. Señor presidente (Aplausos.) [Aznar, 1999] Señor presidente [...] 
En casos como el que sigue, el significado consecutivo se ve acompañado de otro $u$ otros aspectos sintáctico-pragmáticos, por ejemplo la serie enumerativa, con estructura paralelística en estos dos ejemplos:

(24) Señoras y señores diputados, yo no sé si bajar impuestos es de izquierdas, de centro o de derechas, pero me alegro de que ya no estemos tan solos en la defensa de las virtudes de bajar los impuestos $y$ de reducir los tramos. (Aplausos.-Rumores.)

(25) Desde 1996 hemos querido impulsar la libertad, la confianza y la solidaridad como los valores básicos de la sociedad española. En ellos se inspiran las reformas que hemos emprendido y los mismos valores serán los que sigan inspirando la acción política del Gobierno. Este es mi compromiso ante la Cámara y ante todos los ciudadanos españoles. Muchas gracias, señora presidenta. (Fuertes y prolongados aplausos.)

$\mathrm{O}$, en estos otros dos casos, a modo de ejemplos, junto al posicionamiento y la serie enumerativa lineal:

(26) Los españoles confiamos en nosotros mismos y eso es lo que nos permite plantearnos nuevos retos para nuestro futuro. Somos una sociedad más libre, más fuerte, más desarrollada y más decidida a seguir avanzando, y estos creo que son los verdaderos rasgos distintivos del estado de nuestra nación. Muchas gracias, señora presidenta. (Prolongados aplausos.)

(27) Estoy seguro de que los españoles sabrán valorar este empeño. Son millones los españoles que comparten la necesidad de estas reformas. En los resultados ya alcanzados apreciamos el valor de 
continuar en este camino de progreso y de prosperidad. No deja de sorprenderme que algunos persistan en mantener el debate político en términos retrospectivos, intentando resucitar tentaciones de particularismos, exclusiones o insalvables fracturas ideológicas (Aplausos.).

Otros cuatro casos hemos hallado con estructura justificativa, sin conectores explícitos pero con significados que retoman actos anteriores con objeto de rematar el enunciado con el refuerzo argumentativo; en los tres ejemplos siguientes, se acompañan de serie enumerativa, tipo lineal o lista de palabras:

(28) El programa que impulso como presidente del Gobierno nunca ha sido, señorías, un proyecto personal, es el programa de un partido político que ha merecido la confianza mayoritaria de los ciudadanos en las dos últimas elecciones generales. Siempre he estado convencido de que la fortaleza de una democracia la da, más allá de las personas, la seriedad de los proyectos políticos y la solidez de los partidos. (Aplausos.) [Aznar, 2003]

(29) La paz que anhelamos ha de fundarse en la dignidad humana, en la justicia, en el respeto de los derechos y en las libertades individuales. Esa será la paz que tendrá la fortaleza y la voluntad para ser generosa. (Aplausos.) [Aznar, 1999]

(30) Señorías, hay que proyectar, planear y debatir juntos acerca de cómo podemos servir mejor las aspiraciones e intereses de los españoles. Nosotros queremos hacerlo, y ese es el sentido y la razón de toda la obra del Gobierno. Muchas gracias, señor presidente. (Fuertes aplausos.-Rumores.) [Aznar, 1999] 


\section{Y en este otro, de tipo paralelístico:}

(31) El terrorismo está sintiendo las consecuencias de su fracaso. $\mathrm{Ni}$ las mentes más embrutecidas de los terroristas pueden esperar ya la derrota del Estado democrático, ni existe la más mínima posibilidad de que el crimen rinda a nadie beneficios políticos. Ni las estrategias más falaces han conseguido desviarnos de nuestros principios, ni las ofensivas más brutales han minado nuestra voluntad común de acabar con esta lacra. (Aplausos.) [Aznar, 2003]

Finalmente, nos hemos encontrado con la aparición de una reformulación con carácter conclusivo y marcador explícito, dicho de otra manera, y acompañada de contraste:

(32) Los casi nueve millones de declarantes que se encuentran bajo ese umbral obtendrán una rebaja media del 30 por ciento. Más de un millón y medio de contribuyentes quedarán completamente eximidos del pago del impuesto. Los casi cinco millones de españoles con rentas entre dos y cinco millones de pesetas tendrán una rebaja del 11 por ciento y los casi 830.000 que declaran más de esa cantidad tendrán una rebaja del 6,2 por ciento. Dicho de otra manera, los ciudadanos con rentas más altas aumentarán proporcionalmente su contribución al impuesto, mientras que las rentas bajas y medias disminuirán la suya. (Aplausos.) [Aznar, 1998].

\subsection{Contraste}

Curiosamente el contraste ha sido el mecanismo considerado por algunos estudiosos como el más utilizado para la 
propiciación del aplauso; en Atkinson (1984), por ejemplo, aparece en una tercera parte de los casos en que se produce dicho aplauso, en tanto que en un estudio posterior del mismo autor (1985) su uso llega casi a dos terceras partes (63\%). En Aznar, hemos encontrado solo seis apariciones (17.1\% de los aplausos), todas ellas acompañadas de otros mecanismos; en cuatro de esos seis casos se acompaña de dos elementos más, uno de ellos es la serie enumerativa paralelística, mecanismo con el que guarda una cierta relación:

(11) Todos los aquí presentes debemos reflexionar sobre lo que significa y a lo que nos compromete el espíritu de Ermua. Los vascos y el conjunto de los españoles nos lo han dicho con claridad: hay que trazar una línea nítida entre la barbarie y la paz, entre el imperio del terror y el imperio de la ley. O se está con la barbarie o se está con la civilización, pero no es posible tener un pie en un lado y otro más allá de la línea. (Varios señores diputados: ¡Muy bien!Aplausos.) [Aznar, 1998]

Junto al ataque a los rivales, que no respetan el espíritu de Ermua, ${ }^{15}$ Aznar termina con un contraste: "O se está con la barbarie o se está con la civilización, pero no es posible tener un pie en un lado y otro más allá de la línea", contraste que sirve para potenciar una relación semántica conclusiva y ejemplificadora, a modo de remate de la idea. Vemos que dos bloques presentan una estructura paralelística con ele-

\footnotetext{
${ }^{15}$ Se conoce como Espíritu de Ermua un movimiento cívico espontáneo, en contra del terrorismo vasco, surgido tras el secuestro y posterior asesinato a manos de ETA, en 1997, de un concejal del Partido Popular, en la localidad vizcaína de Ermua.
} 
mentos comunes. El segundo miembro repite la disposición del primero, cambiando algunos elementos, pocos, pues estos elementos comunes han de tener una extensión breve y parecida para no perder su eficacia; de este modo, al emitir el orador el primer tiempo a la par que aumenta el énfasis, el espectador ya está a la espera del segundo; en este segundo, acompañado del descenso enfático — tal y como ha indicado para algunos casos del propio JMA, Fernández García (1998: 230) - los últimos golpes de voz se hacen de modo que se dé tiempo al público a adelantarse con el aplauso o a comenzar inmediatamente después del final. Un segundo ejemplo de este tipo es el siguiente:

(33) Me alegro de que ya no estemos tan solos y me alegro también de saber que contaremos con nuevos e inesperados apoyos en los siguientes pasos que vamos a dar en esta materia. Hace tres años bajamos el impuesto sobre la renta. La oposición a esta rebaja fue frontal y llegó hasta el Tribunal Constitucional. Los firmantes de aquel recurso dijeron entonces que establecer un mínimo familiar y rebajar tramos reducía la progresividad del impuesto. Ahora, la mitad de ellos sostiene exactamente lo contrario. A estos les alabo su cambio de opinión, a la otra mitad le alabo su coherencia. (Aplausos.-Rumores) [Aznar, 2001]

Los elementos paralelísticos vuelven a presentarnos sus elementos comunes les alabo su, lo que refuerza la construcción y prepara el aplauso; este se produce antes de que termine el enunciado, exactamente comienza en le alabo. Lo paralingüístico ayuda también a la consecución de dicho aplauso: habla de forma algo más lenta, lo que hace que 
rompa el volumen constante que tanto perjudica su oratoria; la pausa tras materia es amplia y los espacios entre el antes y el ahora se hacen mediante un uso estratégico de las pausas y de la velocidad; tras contrario, realiza una pausa también larga y prepara el paralelismo final con el mismo uso estratégico de la velocidad a lo que suma la entonación ascendente del primer elemento (lo que predispone al aplauso) y descendente del segundo. No podemos decir lo mismo de aspectos kinésicos, pues continúa la inexpresividad tanto en las manos del orador, que va de estar apoyada en el atril con las palmas hacia abajo a tomar los papeles y viceversa, y en cuanto a la cara el mismo movimiento, pero esta vez de los papeles al público, de tarde en tarde.

En el caso siguiente, el contraste entre el ayer y el hoy se ve reforzado por otro recurso, al que ya nos hemos referido repetidamente con anterioridad, la serie enumerativa de ambos tipos:

(34) Hasta hace tres años había miedo de ponerlas en práctica. Hoy vemos los beneficios de la austeridad, de la flexibilización de los mercados, de la reducción de los impuestos, de la disminución del tamaño del sector público; hoy vemos las ventajas de contar con una economía sana y abierta y de lo mucho que favorece a todos la expansión de la libertad. (Aplausos.) [Aznar, 1999]

A partir de hoy vemos dos estructuras paralelísticas, cuya estructura, a la par que potencia el contraste entre el ayer y el hoy, propicia, como todas las series enumerativas, el aplauso: 
Hoy vemos los beneficios

de la austeridad,

de la flexibilización de los mercados,

de la reducción de los impuestos,

de la disminución del tamaño del sector público;

hoy vemos las ventajas

de contar con una economía

sana y

abierta y

de lo mucho que favorece a todos la expansión de la [libertad

Ahora bien, en otras ocasiones, el citado contraste no viene fortalecido por elemento alguno que no sea el meramente ideológico, generalmente entre el antes (cuando gobernaban otros) y el ahora (cuando gobierna el presidente):

(35) Bajar el Impuesto sobre la Renta es ahora posible y no lo fue antes porque, en los primeros años noventa, el desorden de las finanzas públicas generó un creciente e insoportable endeudamiento (Rumores.-Aplausos.) [Aznar, 1998]

\subsection{Posicionamiento}

Es un recurso con el que el político de turno se posiciona en primera persona como una manera de intensificar y cerrar determinadas ideas iniciadas momentos antes. En este sentido, consideramos el término en una de sus acepciones: el que se refiere al punto de vista del hablante en relación a lo que él dice y no en relación a los otros participantes en 
la interacción; decimos esto porque es, precisamente, esta última acepción la que, a través de la lingüística interaccionista de Goffman (1981), más se ha extendido. Si bien los procedimientos empleados para tal 'posicionamiento' son varios, nosotros nos vamos a referir a la primera persona del singular con verbos volitivos que muestran la actitud del sujeto en orden a la realización o deseo de realización de una acción; ambos mecanismos marcan el punto de vista del hablante y con frecuencia aparecen al inicio del cierre de un enunciado, como remate intensificador final.

Así, en el siguiente ejemplo, por si las razones previas no eran suficientes y con objeto de intensificar su capacidad de convicción, Aznar, que lo emplea en siete ocasiones, pasa del nosotros al yo y lo refuerza mediante una serie enumerativa paralelística:

(36) Queremos facilitar a los empresarios sin asalariados la contratación de su primer empleado. Se trata de romper la barrera psicológica de contratar al primer trabajador. Tenemos un gran campo por delante. Existen en España millones de autónomos que trabajan solos y a los que quiero dirigirme de una manera muy singular para decirles que este es el momento de tomar la iniciativa y que esta es la ocasión de asumir nuevos objetivos. (Varios señores diputados: ¡Muy bien!-Aplausos.) [Aznar, 1998]

Los mecanismos no verbales empleados aquí son claros: cambia el volumen de voz a partir de decirles, sus pausas son más largas y la velocidad del habla es menor, con objeto de marcar con claridad las dos estructuras (serie enumerativa) dependientes de la matriz para decirles; todo ello se acompa- 
ña de la fijación de su mirada en el hemiciclo, suspendiendo el movimiento normal (que hubiera sido del hemiciclo al papel). Es más, espera el aplauso algún segundo hasta que este arranca. Sí hay conciencia de búsqueda en esta ocasión. Además, la primera persona y el verbo de voluntad son claros indicadores de un punto de vista compromisivo del acto. Algo parecido encontramos en el resto de ejemplos de este apartado, en los que a diversas razones argumentativas favorecedoras de una idea, de un logro, de una realidad, se suma, como punto de máxima convicción, el posicionamiento explícito del mismo presidente, lo que desencadena el aplauso de los seguidores:

(37) Señorías, hace un año la provisión de la fiscalía de la Audiencia Nacional provocó una polémica que tuvo reflejo en esta Cámara formulándose el reproche de que la decisión adoptada era ilegal. El pronunciamiento del más alto tribunal ha confirmado la legalidad de aquella decisión y es un ejemplo también que debe servirnos para que, entre todos, procuremos preservar cuanto afecta al ámbito de la justicia del uso partidista. Quiero, en todo caso, reiterar mi respeto a la autonomía del ministerio fiscal, en el que depositamos la confianza más plena para el cumplimiento de su papel constitucional de defensa del interés público. (Rumores.-Aplausos.) [Aznar, 1998]

(38) Hemos comunicado al Gobierno marroquí que deseamos mantener unas relaciones diplomáticas fluidas, que sólo pueden basarse en el respeto mutuo. Es imprescindible volver al statu quo anterior a la ocupación de la isla. Hacemos todos los esfuerzos diplomáticos para restaurar la legalidad internacional. España 
no aceptará hechos consumados. Estoy convencido de que ante un asunto como este el Gobierno encontrará el respaldo de la inmensa mayoría de la Cámara. (Aplausos.) [Aznar, 2002]

\section{Conclusiones}

El aplauso, como la incidencia más importante del discurso, no ha tenido la atención, hasta ahora, que merece en la bibliografía internacional. El presente artículo muestra cómo la emisión del aplauso está motivada por determinados elementos que lo propician. En este artículo nos hemos ceñido a las estructuras sintáctico-pragmáticas y hemos concluido con que determinados mecanismos inéditos en la citada bibliografía, como, por ejemplo, el refuerzo conclusivoargumentativo y su tipología (consecutivo, justificativo y reformulador), el posicionamiento o la mención de personajes, instituciones, etc. tienen un importante papel en la propiciación de dicho aplauso. El estudio de estos mecanismos, imbricados siempre con aspectos entonativos, kinésicos o paraverbales, sirve para enriquecer la nómina de otros aspectos hasta ahora considerados por los estudiosos. También es posible, porque la aparición de casos en nuestro corpus así nos lo muestra, una interpretación diferente y más amplia de la serie enumerativa como elemento que predispone al aplauso. Precisamente, es el análisis de componentes hasta ahora inéditos en los reducidos estudios sobre el aplauso lo que aporta el presente artículo y lo diferencia, entre otras consideraciones, de los llevados a cabo hasta el momento. 


\section{Bibliografía}

AtKinson, J. Maxwell (1984): "Public speaking and audience responses: some techniques for inviting applause", en J.M. Atkinson y J.C. Heritage (eds.), Structures of Social Action: Studies in Conversation Analysis, Cambridge, Cambridge University Press, págs. 370-409.

Atkinson, J. MaXwell (1985): "Refusing invited applause: preliminary observations from a case study of charismatic oratory", en T.A. van Dijk (ed.) Handbook of Disourse Analysis, vol. III., Londres, Academic Press, págs. 161-181.

Bull, Peter (2000): "Do audiences applaud only "claptrap" in political speeches? An analysis of invited and uninvited applause", Social Psychological Review, 2, págs. 32-41.

Bull, Peter (2006): "Invited and uninvited applause in political speeches", British Journal of Social Psychology, 45, págs. 563-578.

Bull, Peter (2012): "The microanalysis of political discourse", Philologia Hispalensis 26/1-2, págs. 79-93

Bull, Peter y Merel Noordhuizen (2000): "The mistiming of applause in political speeches", Journal of Language and Social Psychology, 19, págs. 275-294.

Bull, Peter y P. Wells (2002): "By invitation only? An analysis of invited and uninvited applause", Journal of Language and Social Psychology, 21, págs. 230-244.

CoRTÉs, Luis (2005): «La serie enumerativa: cuestiones de partida». En Palabras, Norma, discurso. En Memoria 
de Fernando Lázaro Carreter, Salamanca: Universidad, págs. 365-380.

CORTÉs, LuIs (2015): “Sobre incidencias en los discursos de los debates en torno al estado de la nación. Cuestiones previas", Tonos Digital, 28.

Cortés, Luis, ed., (2008): La serie enumerativa, Madrid: Arco/Libros.

Fernández García, Francisco (1998): "La propiciación del aplauso en la oratoria política: composición y puesta en escena", en Antonio Romero (dir.) Lenguajes y comunicación: consideraciones estéticas y didácticas, Granada, Universidad, págs. 221-233.

Goffman, Erving (1981). Forms of Talk. Oxford: Blackwell. Grady, Kevin y Jonathan Potter (1985): "Speaking and clapping. A comparison of Foot and Theacher's oratory", Language and Communication, 5, págs. 173-183.

Heritage, John y David Greatbatch (1986): “Generating Applause: A Study of Rhetoric and Response at Party Political Conferences”, AJS, 92,1,págs. 110-157.

ILIE, C. (2001). "Unparliamentary language: Insults as cognitive forms of confrontation",

Language and ideology, Vol. II: Descriptive cognitive approaches, R. Dirven, R.

Frank \& C. Ilie (eds.), Amsterdam: John Benjamins Publishing Company, págs. 235-263.

ILIE, C. (2004): "Insulting as (un)parliamentary practice in the British and Swedish Parliaments:A rhetorical approach", Cross-Cultural Perspectives on Parliamentary Discourse,P. Bayley (ed.), Amsterdam: John Benjamins Publishing Company, págs. 45-86. 
RAE (2009): Nueva gramática de la lengua española, 2 volúmenes, Madrid, Espasa Libros.

Roger, D. B., P.E. Bull y S. Smith (1988): “The development of a comprehensive system for classifying interruptions", Journal of Language and Social Psychology, 7, págs. 27-34. 
El aplauso al presidente Aznar

64 Anuario de Letras. Lingüística y Filología, volumen III, 1, año 2015: 25-64 